\title{
DEVELOPING CUSTOMER SERVICE STANDARDS
}

\author{
Venelin TERZIEV, Vanya BANABAKOVA, Marin GEORGIEV \\ "Vasil Levski" National Military University, Veliko Tarnovo, Bulgaria \\ terziev@skmat.com
}

\begin{abstract}
Ensuring effective customer service requires targeted efforts in a number of areas, one of which is to develop service standards for each market segment. Differentiating customer service is achieved by adopting different standards according to the following criteria: availability of assortment varieties and quantities in percentage of orders placed; actually delivered quantities and assortment varieties in percent of the ordered ones; time (cycle) to execute orders in hours or days, and more. The development of service standards uses the results of its analyzes and assessments. Standards relating to customer service should be developed in the following areas: responsibilities of management; quality control system; supplier's obligations; design management; document management; purchase quality; product identification; management of the processes of providing services; customer service quality assessment; management of control, measuring and implementation equipment; corrective actions applied in the case of established discrepancies in the provided services; loading, unloading, storing, packaging, delivery and storage of the product; customer interaction; control over data related to service quality; internal audits related to service quality; personnel training; statistical methods. This paper explores the need and capability to develop customer service standards and provides an algorithm for developing standards for employee behaviour toward customers.
\end{abstract}

Keywords: customer service, standards, custom requirements.

\section{Introduction}

Ensuring effective customer service requires targeted efforts in a number of areas, one of which is to develop service standards for each market segment.

Customer serving standards are defined parameters that have a quantitative expression. They are valid for a longer period of time, they are pre-bid and refer to all customers in a given category (if the organization has adopted a system for categorizing their customers). They facilitate the execution of orders and related procedures. Standards serve to plan the necessary conditions, operations and procedures, as well as to conclude longterm contracts with permanent clients for their service.
According to Naydenov, service standards include a description of the customer's desired service, the operations that staff should perform and the way they are performed. They should also contain criteria for assessing the quality of work of the staff. The evaluation is carried out by comparing the requirements of the standard with the actual results.

The purpose of this study is to investigate the need and capability to develop customer service standards and to propose an algorithm for developing standards related to employee behaviour towards customers. [1-2]

2. Developing customer service standards Differentiating customer service is achieved 
by adopting different standards according to the following criteria:

- availability of assortment varieties and quantities in percentage from orders placed;

- $\quad$ quantities actually delivered and assortment varieties in percentage to the ordered ones;

- time (cycle) to execute orders in hours or days, and more.

The development of service standards uses the results of its analyzes and assessments. Service standardization is an activity of unifying plans, processes, planning system, data exchange system, document turnover, cargo units, commercial and transport packaging, etc. Good practices are regulated in standards (ISO international standards). Standards relating to customer service should be developed in the following areas: responsibilities of management; quality control system; supplier's obligations; design management; document management; purchase quality; product identification; management of the processes of providing services; customer service quality assessment; management of control, measuring and implementation equipment; corrective actions applied in the case of established discrepancies in the provided services; loading, unloading, storing, packaging, supply and storage of the product; customer interaction; control over data related to service quality; internal audits related to service quality; personnel training; statistical methods.

From a methodological point of view, ISO standards answer the question of what needs to be done to ensure a high level of service, and the question of how to do so is the TQM (Total Quality Management) concept. The latter is geared to improving service when a certain level has already been reached and the implementation of ISO 9000 standards is aimed at reducing the likelihood of deviations. As a result, they complement each other in order to achieve a unified goal, but at different stages of the enterprise's business to provide the necessary level of customer service. The development of ISO 9000 and TQM-based methods is linked to the possibility of integrating the two approaches (Table 1).

Table 1. Conceptual elements of ISO 9000 and TQM

\begin{tabular}{|l|c|c|}
\hline \multirow{2}{*}{\multicolumn{2}{|c|}{ Elements }} & \multicolumn{2}{c|}{ Importance for the concept } \\
\cline { 2 - 3 } & ISO 9000 & TQM \\
\hline Leading role of management & Great & Great \\
\hline Creating Workgroups & App. Medium & Great \\
\hline Methods and Instruments & App. Medium & Great \\
\hline Guideline Guidance & Medium & Голямa \\
\hline Official Quality Standards & Great & App. Medium \\
\hline Integrated Quality System & & \\
\hline Employee Involvement, Credentials, Training & Great & App. Medium \\
\hline
\end{tabular}

The development and implementation of standards requires the organization to accurately determine customer service types, the cost of providing alternative services, and measures for measuring and controlling the services provided.

A key element is the analysis of the target market segment, the specific customers, the product (service) / product line, customer service levels, cost, measurement and 
control over the application of the standards.

At the core of the developed and implemented standards is the development and establishment of the customer service policy, which should start with a consumer demand analysis. It must also take into account the interchangeability of products and the number and characteristics of competitors, as this determines not only the relationship between the organization and customers but also its sales. The need to maintain large stocks and / or use additional transport is the greater the lower the degree of interchangeability of products. The organization should fairly accurately determine customer behaviour when needed to fill depleted stocks of specific products as a whole and for the different regional markets.

The consequences of stock depletion are not the same for all customers. They are to a large extent determined by the size of the customer and / or the volume of supplies. Losses in such cases are much greater when dealing with large customers and large deliveries, and this proves to be sufficient basis for implementing a different service policy. In this context, it is often practiced to divide customers by importance into different groups using the $\mathrm{ABC}$ analysis method. In the process of conducting these analyzes, specific customer requirements (eg, specific delivery dates) need to be precisely established to accommodate the service. Service policy should be tailored to the current competitive level of service on the market, as lower-level tactics places the organization at a disadvantage. However, this also depends on its ability to provide better service at costs that will not significantly affect price competitiveness.

Defining the level of customer service should allow for quantitative measurement because tasks such as „ninety percent customer satisfaction" that are pledged in service policy are not easily measurable and therefore difficult to achieve and control. The ambiguous and not easily quantifiable policy does not provide opportunities to evaluate and control the activities and costs of customer service. In this respect, quantifiable and traceable indicators and targets for each customer service element should be formulated and quantified in service standards, which can be in the following directions:

- Product availability - usually measured in terms of saturation with a specific product (stock availability percentage) or order execution (stock performance percentage). In the standard for this element, time availability can also be combined to make a measure, such as "ninety-five percent of orders to be delivered on time and in full". Product availability is a critical element of service. Therefore, it is recommended that the company has standards for the number and percentage of correctly executed orders over a certain period of time.

- Order cycle length - It is one of the most frequently treated elements in service standards. It is usually formulated as a percentage of orders executed for a certain number of days after receipt - for example, "ninety-five percent of deliveries made within three days of receipt of the order". To determine the compliance of time standards with customer needs, managers plan cumulative distributions on actual delivery times and compare them with policy-based policies - for example, if $90 \%$ of deliveries within the three days of receipt of the order, the market and customer information for orders received is analyzed to determine how many orders were delivered on time and how much later.

- Flexibility of the logistics system Defined in service standards as an organization's ability to respond to special customer needs - for example, organizing very fast shipments when needed. In such cases, tasks are specified in the relevant standard, such as ,95\% feasibility of fast orders in accordance with customer instructions".

- The information security of the logistics system - It is extremely important for 
customers. In this regard, the company can formulate its information policy in a relevant standard based on user requirements. The availability of such a standard also facilitates the process of controlling the quality of information of the logistics system.

- Miscarriages in the logistics system - The organization should be able to control and quickly eliminate any deviation with a negative effect on the customer - for example, if a shipment is damaged and can't be used by the customer. Reposting required products requires extra time and is associated with additional costs, which reduces service levels and increases costs. The situation is similar in the case of incorrect invoices as it makes it difficult to establish the amount due.

- After sales support and service - It is an important part of the development of logistics - for example, equipment assembly, complete supply of spare parts and others. In this direction, standards should be set, mainly for the time of delivery of the required spare parts, by formulating real measurable tasks, eg "95\% of all requested spare parts will be delivered on the day of the order".

In the practical realization of the standardization of the customer service there are also some features:

First, the parameters set in the standards should be realistically achievable but not too low. It is necessary for the standard to contribute to the effective realization of the existing and potential competitive advantages of the organization.

Second, it is often recommended that service standards be pursued at a level of $100 \%$. This should only be perceived as a philosophy and an ideal, since setting levels below $100 \%$ do not stimulate improvements.

Thirdly, it is advisable that the organization's policy and standards of service be formed and established in close interaction with customers through consultation with them. Practice shows that the best approach is that organizational policy and service standards are open to customers.

Fourthly, the development of procedures for measuring and controlling levels and standards of customer service is a basic prerequisite. Using techniques such as statistical methods of control, maintaining feedback, and undertaking corrective actions are essential to success. If customer service standards are ineffective, the organization should not hesitate to correct or remove them.

It should also be noted that the procurement of raw materials of organizations, as a whole, necessitates reaching almost one hundred percent of service levels because costs related to the depletion of commodity stocks are almost always less than the costs of exhausting stocks of raw materials. In such situations, opportunities are usually sought for replacing raw materials with others. Often, however, the following is observed:

- The short periods for carrying out specific production activities do not allow them;

- A number of raw materials are strictly specialized and specific, their suppliers are limited and replacements are impossible;

- Specific technical and technological requirements make it very difficult for trouble-free replacement.

In this sense, any breach of supply activities may have significant negative consequences for customers (manufacturing enterprises), especially if the depletion of stocks is prolonged. Obviously, the cost of depletion of supply stocks is very high, which requires additional effort on the movement and storage of raw materials. The depletion of stocks of raw materials is linked to causing multiple negative internal and organizational effects - mainly triggering a chain reaction, as the depletion of stocks of raw materials in an enterprise creates problems for the others in the technological chain. [1, 3-4] 
3. Order for implementation of developed standards:

- Conduct meetings with responsible persons to explain the need for strict compliance with the established standards by the employees of the organization. Explain the essence of innovation, give instructions for the actions of those responsible, clearly outline the sphere of their responsibilities, the level of authority and the resources they can dispose of in their tasks. Answer all questions.

- Some points in customer service standards may be added.

- The result should be focusing on the ability to work with clients in line with accepted standards.

On the basis of this study, the following conclusions are highlighted:

- Standards relating to customer service should be developed in the following areas: responsibilities of management; quality control system; supplier's obligations; design management; document management; purchase quality; product identification; management of the processes of providing services; customer service quality assessment; management of control, measuring and implementation equipment; corrective actions applied in the case of established discrepancies in the provided services; loading, unloading, storing, packaging, supply and storage of the product; customer interaction; control over data related to service quality; internal audits related to service quality; personnel training; statistical methods.

- The basis for the developed and implemented standards is the development and establishment of the customer service policy, which should start with the analysis of consumer demand. It must also take into account the interchangeability of products and the number and characteristics of competitors, as this determines not only the relationship between the organization and customers but also its sales.

- Quantifiable and traceable metrics and targets for each customer service element should be formulated and embedded in service standards.

- In developing service standards, it is appropriate to apply an algorithm that focuses primarily on employee behaviour standards for customers, which includes four stages with the appropriate tasks and order for developing the standards.

In conclusion, ensuring quality customer service requires the development and deployment of standards that require the organization to accurately determine customer service levels, the cost of providing alternative services, and the measurement and control measures for the services provided. [5]

\section{Conclusions}

In conclusion, ensuring quality customer service requires the development and deployment of standards that require the organization to accurately determine customer service levels, the cost of providing alternative services, and the measurement and control measures for the services provided. [6]

\section{References}

[1] Terziev, V., Banabakova, V., Georgiev, M., Customer service standards, Proceedings of INTCESS 2018- 5th International Conference on Education and Social Sciences 5-7 February 2018- Istanbul, Turkey, 2018, pp. 431-437.

[2] Terziev, V., Banabakova, V., Georgiev, M., Possibilities for determining customer's profitability, Proceedings of INTCESS 2018- 5th International Conference on Education and Social Sciences 5-7 February 2018- Istanbul, Turkey, 2018, pp. 438-444. 
[3] Terziev, V., Banabakova, V., Stefanov.S., Georgiev, M., Study of consumer behaviour, Fifteenth International Scientific Conference: Knowledge in practice, Bansko, Bulgaria, International Journal of Knowledge and Learning, Institute of Knowledge ManagementSkopje, Vol. 20, N1, pp.465-471, 2017.

[4] Terziev, V., Banabakova, V., Stefanov, S., Georgiev, M., Evolution of the marketing concept, marketing mix -,,mix of components“ or a set of actions, Fifteenth International Scientific Conference: Knowledge in practice, Bansko, Bulgaria, International Journal of Knowledge and Learning, Institute of Knowledge ManagementSkopje, Vol. 20, N1, pp. 213-218, 2017.

[5] Terziev, V., Banabakova, V., Marketing, Institute of Knowledge Management, Skopje, Macedonia, p. 107, 2017.

[6] Terziev, V., Banabakova, V., Georgiev, M., Customer's Profitability Analyses and Customer Service Policies, Journal of Innovations and Sustainability, Innovations and Sustainability Academy, Vol. 3, N 3, pp. 39-48, 2017. 